\title{
Candidate Technologies for High-Capacity Optical Communication Systems
}

\author{
Lidia Galdino, Daniel Semrau and Polina Bayvel \\ Optical Networks Group, Dept. of Electronic \& Electrical Engineering, UCL (University College London), UK \\ l.galdino@ucl.ac.uk
}

\begin{abstract}
:
The practicalities in designing high-capacity optical communication systems are described. With a given perspective on the present and future technologies, we cover the transceiver design and optical amplifier technologies to maximize optical fiber capacity.
\end{abstract}

OCIS codes: (060.0060) Fiber optics and optical communications; (060.2360) Fiber optics links and subsystems

\section{Introduction}

A key aim when designing a new optical transmission system is to increase the amount of data sent for a given cost. It can be achieved by maximizing the spectral efficiency (SE), as well as expanding the usable optical fiber bandwidth. Over the last few years the research community have focus on maximizing the channel spectral efficiency with advanced coding and modulation techniques for approaching Shannon limit [1]. Ultimately, all proposed methods must be applied to higher-order modulation formats to accomplish higher SE. However, the higher modulation format, the higher signal-to-noise ratio (SNR) requirements [2]. Therefore, a key condition to maximizing the number of information bits that can be reliably transmitted over a fiber channel relies on maximizing the SNR achieved at the receiver.

In an optical fiber system, an upper bound on the achievable SNR and therefore data throughput, is set by the noise introduced by the transceiver subsystems. The achievable SNR is then further limited by the nonlinear signal distortion, inherent to transmission through an optical fiber and amplified spontaneous emission (ASE) noise, generated by in-line optical amplifiers. Therefore, the received SNR ( $\left.\mathrm{SNR}_{\text {Total }}\right)$ can be expressed as $\mathrm{SNR}_{\mathrm{Total}}^{-1}=\mathrm{SNR}_{\mathrm{TRX}}^{-1}+\mathrm{SNR}_{\mathrm{NLI}}^{-1}+\mathrm{SNR}_{\mathrm{ASE}}^{-1}$ where, $\mathrm{SNR}_{\mathrm{TRX}}, \mathrm{SNR}_{\mathrm{NLI}}$ and $\mathrm{SNR}_{\mathrm{ASE}}$, originates from transceiver noise, fiber nonlinearity and amplifier noise, respectively.

In this work the potential limitations and practicalities to increase the SNR, and therefore data throughput is described. In particular, the impact of different transceiver architectures on the overall transmission system performance is experimentally and theoretically investigated. The transmission system under test is a fully loaded, continuous $90 \mathrm{~nm}$ bandwidth using hybrid Raman-EDFA amplifiers and $70 \mathrm{~km}$ fiber spans. Finally, the trade off between, complexity, performance, capacity and cost is discussed.

\section{Recent progress on transceiver architectures an its impact on transmission capacity}

Due to the evolution of CMOS technology, each new generation of commercial transceiver supports increased channel bandwidth from 28 to $66 \mathrm{GBd}$, and $96 \mathrm{GBd}$ is expected for the next generation of coherent transponders. However, to date, no comprehensive analysis have been carried out for short/medium distances, where the system performance is dominated by the transceiver subsystem constrained signal-to-noise ratio (SNR) [3].

The upper limit on the achievable SNR in a transceiver subsystem is mainly due to the resolution of the digital-toanalog converter (DAC) and analog to digital converter (ADC). The SNR of an ideal DAC / ADC is defined by the number of bits which sets the quantisation noise floor. In practical converters, other distortion sources will add to this noise floor, leading to an effective number of bits (ENOB) lower than the stated bit resolution. Additionally, mainly due to the clock jitter, the ENOB is a function of frequency and is reduced at high frequencies, which consequently diminishes the SNR as the channel symbol rate is increased. A full detailed analysis of the impact of channel symbol rate on transceiver capacity can be found in [3].

The aim now is to further investigate the impact of transceiver constrained-SNR after optical fiber propagation, when linear noise of the optical amplifier and nonlinear interference noise due to a fiber nonlinearity, are added to the signal. The transmission system under investigation is a fully loaded $11.25 \mathrm{THz}$ 
bandwidth using hybrid Raman-EDFA amplified. A full detailed transmission line setup can be found in [4]. On the transmitter side, four carries with 30, 40, 60 , and 85 GBd signals were tested. For each channel symbol rate, $30.5,40.5,60.5$, and $85.5 \mathrm{GHz}$ channel spacing were used, respectively. As illustrated in Fig.1, the modulated signals, carrying DP-256QAM signals, were combined with $11.25 \mathrm{THz}$ of continuous spectrally-shaped amplified spontaneous emission (SSASE) noise used to emulate interfering channels.

For this WDM test-bed setup, the back-to-back $\mathrm{SNR}_{\mathrm{TRX}}$ measured was 21.4, 21.1, 19.5 and $17.1 \mathrm{~dB}$, respectively; a decrease on the $\mathrm{SNR}_{\text {Total }}$ of $4.3 \mathrm{~dB}$ when the channel symbol rate is increased from $30 \mathrm{GBd}$ to $85 \mathrm{GBd}$. Can be observer in Fig.2 that, after increasing the transmission distance to $630 \mathrm{~km}$, the reduction in $\mathrm{SNR}_{\text {Total }}$ decreased to $3.5 \mathrm{~dB}$ (from $19.7 \mathrm{~dB}$ to 16.2 $\mathrm{dB}$ ) for an increase in the channel symbol rate from 30 to $85 \mathrm{GBd}$. This reduction of the delta SNR (among the different channel symbol rates) with the increase of the transmission distance is because the system performance becomes more dominated by the amplifier and fiber nonlinearity noise power at longer distances; this diminishes the impact of transceiver $\mathrm{SNR}_{\mathrm{TRX}}$ on the overall system performance. If the transceiver subsystem was ideal $\left(\mathrm{SNR}_{\mathrm{TRX}}=\infty\right)$, the $\mathrm{SNR}_{\text {Total }}$ would be (approximately) symbol rate independent as the ASE

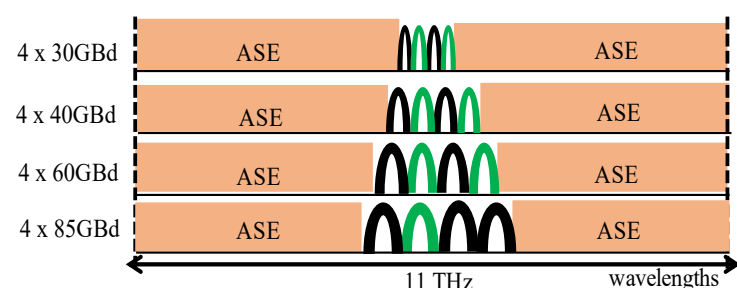

Fig.1: Schematic of different transmitted signals.

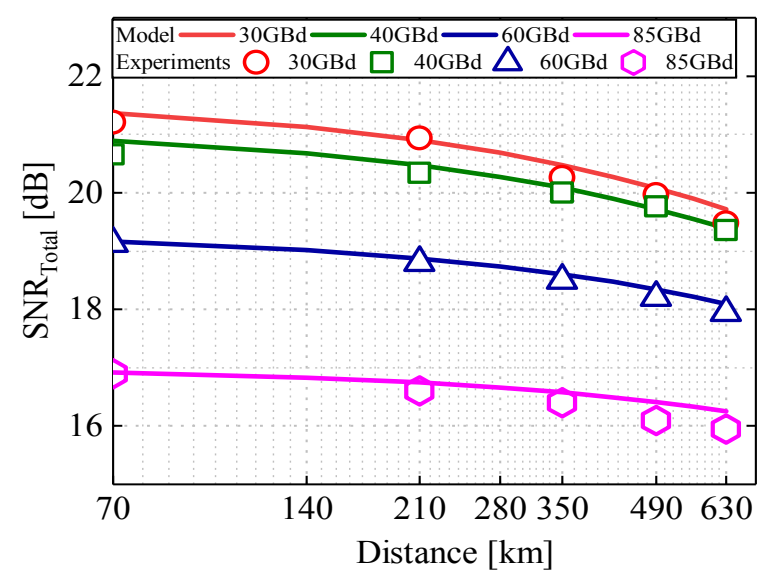

Fig.2:Received SNR as function of transmission distance for different channel symbol rates. The lines are the ISRS-GN model [5] prediction and the markers are the experimental data.

noise power is the same (total power of $22 \mathrm{dBm}$ was maintained for all studied case), and variation on the NLI noise power is negligible for the studied symbol rates [6]. Therefore, the differences in $\mathrm{SNR}_{\text {Total }}$ is fully attributed to the differences in SNR $\mathrm{TRX}$.

Despite this reduction of SNR and therefore the overall system capacity, it should be noted that by using a higher symbol rate per channel, each transceiver's net data rate increases. For instance, after $630 \mathrm{~km}$, each single channel transceiver with 30, 40, 60, and $85 \mathrm{GBd}$ delivered an upper bound on the net data rate of 366, 484, 672 and $850 \mathrm{Gbit} / \mathrm{s}$, respectively. This net data rate was obtained by multiplying the channel bandwidth by the net achievable information rate (which was measured using mutual information (MI) as metric [2] for each studied case). Furthermore, using a higher symbol rate per channel requires fewer transceivers in a given transmission bandwidth. As an example, for this transmission system with $11.25 \mathrm{vTHz}$ bandwidth and $0.5 \mathrm{GHz}$ spacing between the channels, would required $369 \times 30 \mathrm{GBd}$ transceiver. The required number of transceivers would reduce to 277,186 , and 131 for 40 , 60, and $85 \mathrm{GBd}$ signals, respectively. In other words, $85 \mathrm{GBd}$ signals would require $65 \%$ fewer transceivers than the $30 \mathrm{GBd}$ transmission case, and deliver higher data rates per channel. However, it comes with the disadvantage of reducing the total transmission system capacity, as the SNR and MI reduces with the increasing in the channel symbol rate.

\section{References}

1. C.E. Shannon, "A Mathematical Theory of Communication,“" The Bell System Technical Journal Bell 27, 1948.

2. R. Maher, et al.: "Increasing the information rates of optical communications via coded modulation: a study of transceiver performance," Scientifc. Reports, 6, 2016.

3. L. Galdino, et al.: "The Trade-off Between Transceiver Capacity and Symbol rate," OFC, M2E.6, 2018.

4. M. Ionescu, et al.: "90 nm C + L Hybrid Distributed Raman/Erbium-Doped amplifier for High Capacity Subsea Transmission," ECOC, 2018.

5. D. Semrau, et al.: "The Gaussian Noise Mode in the Presence of Inter-Channel Stimulated Raman Scattering," J. Light. Tech., 36(14), 2018.

6. P. Poggiolini, et al.: "Analytical and Experimental Results on System Maximum Reach Increase Through Symbol Rate Optimization,” J. Light. Tech., 34(8), 2016. 